\title{
Traumatic Abdominal Wall Hernia: A Case Report
}

\author{
Somendra Bansal $\cdot$ Kailash C. Vyas
}

Received: 29 January 2010 / Accepted: 30 March 2010 / Published online: 16 January 2014

(C) Association of Surgeons of India 2014

\begin{abstract}
Traumatic abdominal wall hernias are rare injuries despite the high incidence of blunt abdominal traumas. The mechanism of this injury includes a sudden increase in intra-abdominal pressure and extensive shear forces applied to the abdominal wall. We report a case of traumatic hernia of the anterior abdominal wall in a 42-year-old woman presented with blunt injury of the upper abdomen. She was attacked by a bull. She had a clinically evident abdominal fascial disruption with intact skin and was hemodynamically stable. The presence of localized pain, bruising and a reducible swelling or a cough impulse suggested the diagnosis. An emergency mesh repair of the defect was performed, and she recovered well.
\end{abstract}

Keywords Traumatic abdominal wall hernia $\cdot$ Primary closure

\section{Introduction}

Traumatic abdominal wall hernia remains a rarely reported event despite the high prevalence of blunt abdominal trauma $[1,2]$. A review of English literature on this subject showed approximately 50 reported cases since the first report in 1906 [1]. Not surprisingly, many cases have been reported in children, given their weaker parietal wall and more elastic skin [3]. The mechanism of this injury includes a sudden increase

This article was presented as a competitive paper in the ASIRAJ-2010 held at Udaipur (Rajasthan) on 9th-10th January, 2010.

\footnotetext{
S. Bansal $(\bowtie) \cdot$ K. C. Vyas

Department of Surgery, R.N.T. Medical College and Associated group of Hospitals, Udaipur, Rajasthan 313001, India

e-mail: shiva_0102@rediffmail.com
}

in intra-abdominal pressure and extensive shear forces applied to the abdominal wall. The typical location is found at anatomic weak areas in the lower abdomen. We report a case of adult with traumatic abdominal wall hernia with a brief review of the literature.

\section{Case Report}

A 42-year-old woman was brought to the surgical emergency room $6 \mathrm{~h}$ after being attacked by a bull. The animal had stuck her with its horns over her upper abdomen just left to midline. The patient complained of pain and swelling over the involved area. On examination, her vital signs were normal. There was no associated head, chest, pelvic, or limb injury. A bruise and a visible, ill-defined swelling over epigastrium were seen (Fig. 1). On palpation, the area was tender, and swelling decreased on gentle pressure, and cough impulse was present. A defect of $10 \mathrm{~cm}$ in maximum diameter could be palpated with irregular, ill-defined margins. The patient was hemodynamically stable, and the diagnosis was clinically apparent, a midline laparotomy was performed. An irregular defect was found involving abdominal muscles and peritoneum (Fig. 2) and was repaired with an onlay mesh. There was no associated intra-abdominal injury. The patient's recovery was uneventful, and drain was removed on the second postoperative day and was discharged on the third postoperative day.

\section{Discussion}

Traumatic abdominal wall hernia (TAWH) is produced by a sudden application of blunt force that is insufficient to 


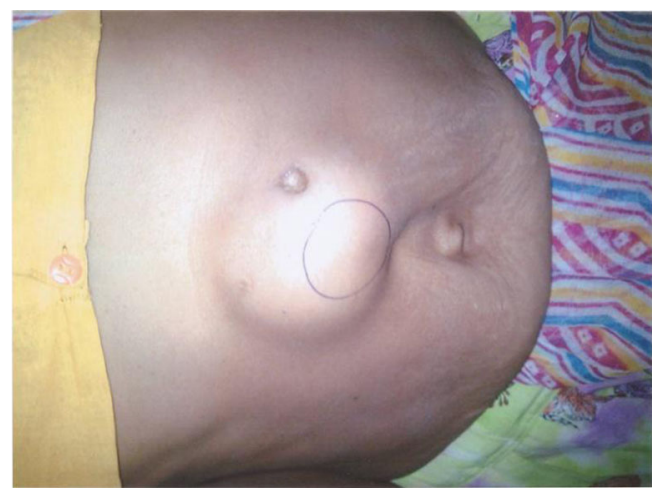

Fig. 1 A bruise and a visible, ill-defined swelling over epigastrium

penetrate the skin but strong enough to disrupt the muscle and fascia [4]. TAWH can be defined as posttraumatic when it appears immediately after injury with evident signs of trauma at the initial presentation and the absence of peritoneal sac and skin penetration [5]. Many mechanisms have been thought to cause traumatic abdominal wall hernias. The most common appears to be bicycle handlebar injury, especially in children [2, 3]. Wood et al. [2] attempted to classify these mechanisms into three types, namely, (1) a small abdominal wall defect caused by low-energy trauma such as bicycle handlebar injuries, (2) a larger abdominal wall defect caused by high-energy injuries such as motor vehicle accidents, and (3) intra-abdominal herniation of the bowel caused by deceleration injuries.

In our patient, the hernia was clinically apparent with a palpable lump, but no associated injury was seen. So, we took the patient in the emergency operation theater without performing a CT scan. CT scanning is the most accurate diagnostic tool for TAWH and should be used for detection of associated intraabdominal injury [4]. There are controversies regarding the timing of exploration: immediate versus

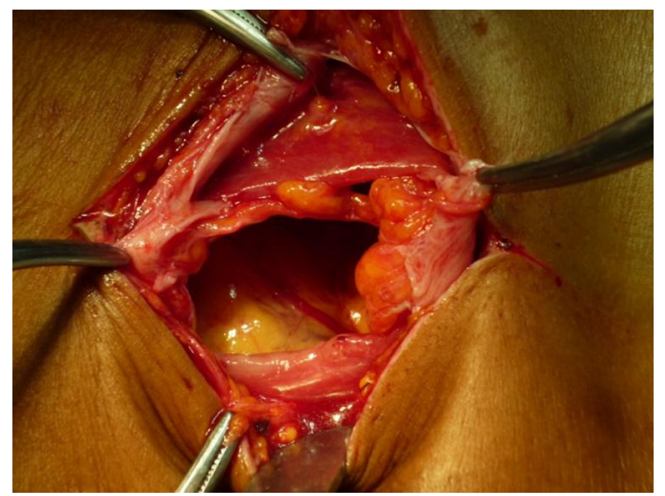

Fig. 2 Irregular defect involving abdominal muscles and peritoneum delayed exploration. Delayed exploration as well as delays in diagnosis can lead to some problems such as bowel strangulation [6] and excessive tension in the primary closure of the defect [7]. Immediate exploration and repair, however, has generally been accepted as a more favorable choice in the treatment of TAWH $[1,2,6]$.

Both mesh repair as well as primary repair have been successfully performed for treatment of traumatic hernia [3]. Low velocity injuries lead to less tissue necrosis and a mesh can be used. When high velocity injuries are present as in motor vehicle accident, mesh may be avoided because of the high risk of infection, unless there is greater tissue loss [1].

\section{Conclusion}

Following blunt abdominal trauma, particularly high-velocity injuries, a high index of suspicion must be reserved for parietal wall swellings, as missed hernias in this setting have a high risk of strangulation [8]. Rectus sheath hematoma and flail chest should be excluded by CT scan. We recommended careful abdominal examination in every patient for fascial defects or reducible swellings. Immediate surgical repair with primary closure of the tissue with prosthetic material is recommended, as muscular disruption prevents simple repair. Since complications due to incarceration or strangulation may occur, delayed or secondary repair is not recommended.

\section{References}

1. Lane CT, Cohen AJ, Cinal ME (2003) Management of traumatic abdominal wall hernia. Am Surg 69:73-76

2. Wood RJ, Ney AL, Bubrick MP (1988) Traumatic abdominal hernia: a case report and review of the literature. Am Surg 54:648-651

3. Mancel B, Aslam A (2003) Traumatic abdominal wall hernia: an unusual bicycle handlebar injury. Pediatr Surg Int 19:746-747

4. Damschen DD, Landercasper J, Cogbill TH, Stolee RT (1994) Acute traumatic abdominal hernia: case reports. J Trauma 36:273-276

5. Clain A (1964) Traumatic hernia. Br J Surg 51:549-550

6. Mahajna A, Ofer A, Krausz MM (2004) Traumatic abdominal hernia associated with large bowel strangulation: case report and review of the literature. Hernia 8:80-82

7. Martinez BD, Stubbe N, Rakower SR (1976) Delayed appearance of traumatic ventral hernia: a case report. J Trauma 16:242-243

8. Agrawal N, Kumar S, Joshi MK, Sharma MS (2009) Traumatic abdominal wall hernia in two adults: a case series. J Med Case Rep $3: 7324$ 